\title{
Digital Business Ecosystem Tools as Interoperability Drivers
}

\author{
Miklós Herdon ${ }^{1}$, Mária Raffai ${ }^{2}$, Ádám Péntek ${ }^{1}$, and Tünde Rózsa ${ }^{1}$ \\ ${ }^{1}$ University of Debrecen, H-4032 Debrecen Böszörményi út 138., Hungary \\ ${ }^{2}$ Széchenyi István University, H-9026 Győr, Egyetem tér 1., Hungary
}

\begin{abstract}
Today's businesses form a highly interconnected network of companies, organisations, technologies, consumers, products and services. The concept of the Digital Business Ecosystem (DBE) has been come life in order to build an Internet-based environment in which businesses will be able to interact with each other more efficiently. In a Digital Ecosystem, a leadership structure is formed in order to be capable of responding to the dynamic needs of the environment. The agent in a Digital Ecosystem can both be a client and a server at the same time. With the same message, agents can simultaneously offer a service to others as a Server and request help as a Client. Industries may have their own systems but they are not yet business ecosystems. Digital ecosystems rely on a technological infrastructure to mediate the formalisation of knowledge in SME networks, the creation of software services, and different type of interactions between SMEs. There are many fields of application in the agro-food industry where interoperability of information system would be essential. Amongst others food tracking and tracing, logistics, SRM and CRM integration all need interoperability solution in a heterogeneous environment. Our prototypes can help in building these cooperative information systems.
\end{abstract}

Keywords: business ecosystem - enterprise information systems - SME.

\section{Introduction}

The Digital Business Ecosystem (DBE) is attainable technological solution for business ecosystems. The concept of DBE was first realized in Europe in 2002. Today there is a progress in European approach towards Digital Ecosystems and currently there are many important ongoing projects related to this in Europe. The concept of Digital Business Ecosystem (DBE) was invented to build an Internet-based environment in which businesses will be able to interact with each other more efficiently. In the near future with the realization of this platform Small and Medium Enterprises (SMEs) will have the chance to compete on the market with the same prospects, regardless of the size and the distance of the city from their businesses [3]. The DBE is supported by new hardware and software technologies as well as network topologies. An Open Source and component-based software, the collaborative environment, development and the popular and quick developing network technologies can help in establishing an extensive use of DBE [2]. 
In a Digital Ecosystem, a leadership structure is formed in response to the dynamic needs of the environment [4]. An agent in a Digital Ecosystem can be both a client and a server at the same time. With the same message, agents can both offer a service to others as a Server and request help as a Client without interfere. In this system there is no centralized control structure or fixed role assignment as there is no preconfigured global architecture, in which communication and collaboration are based on swarm intelligence. Unlike in traditional environments, digital ecosystems are selforganizing systems with the capability of forming different architectural models through swarm intelligence, where local interactions between agents determine the global actions. Occasionally, intelligent agents or entire species may as well configure themselves into a hierarchical organization where the communication channels are defined with a leading agent.

Industries may have their own systems which, however, are not yet a business ecosystem since a business ecosystem has three characteristics as it transfers the business strategies from single co-work to synergic and systematic cooperation (the first characteristic), from product competition to platform competition (the second characteristic), and from single growth to co-evolution (the third characteristic).

Digital ecosystems are based on a technological infrastructure, which mediate the formalisation of knowledge in SME networks, as well as the creation of software services, and B2B interactions between SMEs. The realisation this has by now become commonplace and is in fact provided the initial motivation for CRM, SRM and ERP systems.

In general, ERP systems ensure the integration of the suppliers and buyers (clients) into the business' own systems (communication). However, in case the companies used different ERP systems (from different vendors) in order to get on-line (real-time) connection they required special developments. Small and medium-sized enterprises which have implemented information system (ERP standard created by different developers), recognised that this system does not provide them with interoperability. The DBE concept to achieve the particular case of SMEs may give benefits and ensures the appropriate use of DBE Toolset can provide interoperability. Our goal was to facilitate the implementation of the DBE concept modules, which allows existing systems to get access to the common marketplace more easily.

With our prototype solution the SMEs can provide safe, transparent, direct services to potential customers. With the Client-Server architecture, all data are stored on a dedicated server which can provide great advantages for the server's owner. Our vision is based on P2P and SOA technology and all components are written with Open Source tools [13].

\section{Needs for DBE}

The Digital Business Ecosystem is an evolutionary, self-organized system, which can contribute to the sustainability of the local and regional development through a welldefined software platform [14]. The DBE works just like a natural ecosystem. It is self-organized and able to learn become more efficient. From the Information Technology point of view, a Digital Business Ecosystem is a structure of distributed opensource packages and mashups, based on the Internet ensuring an optimum, stimulating and competitive business development environment, in which all companies, 
especially Small and Medium Enterprises, can cooperate and develop their business affairs [16].

The idea of the Digital Business Ecosystem is "to create an integrated, distributed pervasive network of local digital ecosystems for small business organizations and for local e-governance which cooperates exchanging dynamically resources, applications, services and knowledge." [9]. The aim of the Digital Business Ecosystem (DBE) is to overcome existing barriers and to promote innovative forms of software creation, knowledge sharing and community building, thereby enabling long-term growth and, competitiveness of the European SME sector. As envisioned by [9], the DBE is intended to foster new and flexible ways of co-operation and networking through a dynamic aggregation and self-organizing evolution of services and organizations by means of open-source methods of software and service creation.

Moore first introduced the concept of a business ecosystem as a strategic plan [8]. Moore indicated that organisations form a part of a business ecosystem and as such they should be viewed as a collective rather than an individual entity. In a business ecosystem that crosses a variety of industries, organisations cooperate, compete, and co-evolve capabilities around a new innovation, support new products, satisfy customer needs, and eventually incorporate the next round of innovation.

According to Nachira [10], the key elements of a business ecosystem (top-most layer) include 1) governance, regulations and industrial policy, 2) human capital, knowledge and practices, 3) service and technical infrastructure, and 4) business and financial conditions.

The idea of a technology (or industrial) ecosystem has been used to describe relationships between technologies and organizations. As an example, consider the specific ecosystem view, which had laptop computers as the focal technology and the wireless networking capabilities as the context. The framework is based on a laptoprelated Wi-Fi technology ecosystem. Another more comprehensive demonstration of the analitical approach is an ecosystem model of technology evolution facilitates by focusing on the digital music industry.

\section{Functionality of Enterprise Information Systems}

Integrated information systems under large-scale company conditions have become widespread tools over the past few decades [7]. Information, data management and systematic information derived from these data and arranged according to needs, however, are required by not only large-scale companies but also by small and medium-sized enterprises. This need that was also recognised by the staff developing the ERP system and successfully and as a consequence appeared on the market with a range products developed especially for SMEs.

Different technologies and solutions, such as SOA (Service Oriented Architecture) provide opportunities for further technological and content-related (functional) extensions and assist the development and spread of new business models (SaaS - Software as a Service) [5]. We also conducted an important part of our research while getting to know and analysing ERP suppliers. It was also an objective of ours to get to know the situation of supply in Hungary and to look out on the market in abroad. We got to the conclusion that the market of ERP, as other markets, is characterised also by globalisation and based on the number of its implementation the solutions in Hungary are developed by well-known international market leaders (SAP, Microsoft, ...). The 
analysis of the supply and demand of the markets of ERP systems has a major effect on the part of this work which is dealing with the constructing of a functional system of requirements.

In our research we made survey on using ERPs in SMEs. Approximately 600 requests to fill in the questionnaire were sent out by e-mail or by post. The rate of the response to this request was $16 \% .96 \%$ of the respondents come from small and medium sized businesses. The processing of the questionnaires sent back revealed that $45 \%$ of the respondents used integrated ERP systems and $43 \%$ of them indicated that they used standalone systems while the ratio of the ones using both integrated ERP and standalone systems as well was $4 \%$. The remaining $12 \%$ do not use and do not plan to implement any information systems. As for the developers of the systems $39 \%$ of the respondents credited Hungarian developers with the system while 14\% thought they were of foreign developments. Of the foreign developments SAP, AXAPTA, Microsoft, Abas were named while Cobra, Topinfo, Agroorg were Hungarian developers.

In order to get a more accurate picture of the technological preparedness of the meat industry, we found it necessary to examine the circumstances of the European food industry in general, from the aspects of its use of information and communication technologies (ICT). We conducted our examinations on the basis of the database of e-Business W@tch research.

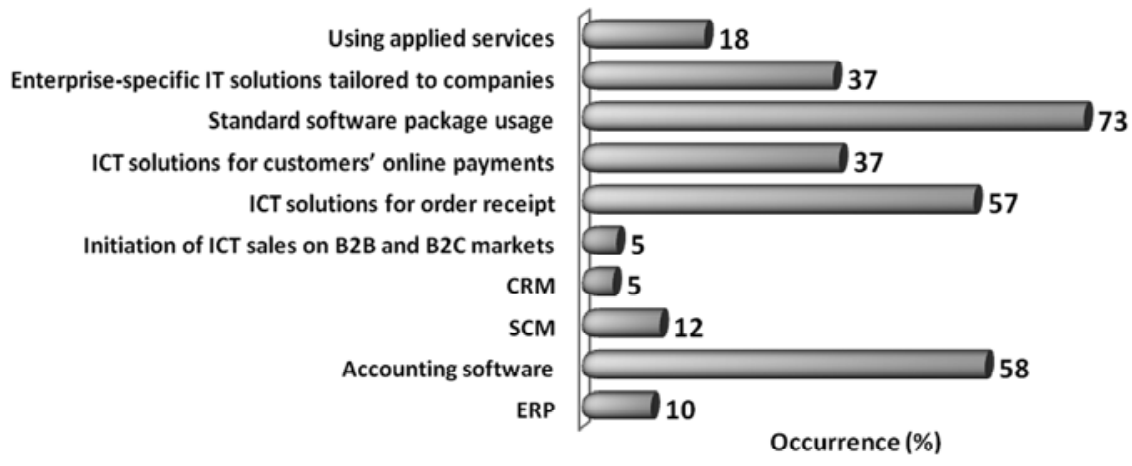

Fig. 1. Ratio of using software in the food sector (2006)

It is clearly shown from the results that the majority of food industry companies already have Internet access, most of which are of-broadband connections. Nevertheless, only $38 \%$ of companies have their own websites and the ratio of those with electronic connection to their suppliers and customers is even lower. As for the applied information system, we can assume that almost three quarters of the enterprises use some kind of standard software packages. The number of accounting software products and systems which are for receiving orders is high also: their ratio is around $60 \%$. Nevertheless, the number of CRM and integrated corporate management systems is rather low, whereas the proportion of software products applied for the initiation of ICT sales is also rather moderate on B2B and B2C markets (Fig. 1.). 
This figures show that the internet access penetration relatively good, the e-cooperation level is very low. We have to make effort for improving this ability.

\section{Interoperability in a Business Environment}

The interoperability focuses on inter-enterprise distributed business processes and flows. According to IEEE's definition [5], interoperability represents "the ability of two or more systems or components to exchange information and to use the information that has been exchanged". These interoperability solutions have of both information integration and application integration. Application integration (comprising message, process, transport and interface) represents the technological solution, while information integration refers to the linguistic, social and philosophical solution (comprising data, context, ontology and interpreter). By integration we mean the cooperation of processes and applications at the event and message levels while multiple systems become one logical unit [1].

Collaborative business requires reliable exchange of data commercial, financial and technical ones as well. Legacy ERP, SCM, LCM and CRM enterprise applications generally manage information which is required for the collaboration, but the software itself is for the most part realised and programmed to be run within specified organizational boundaries.

These systems would allow interoperability between enterprise models as it would facilitate the customisation of suitable software. Enterprise software tools like Enterprise Resources Planning (ERP), Supply Chain Management (SCM) or Customer Relationship Management (CRM) are today's strategic investments for all types of companies. However one should bear in mind that the implementation of such Enterprise Software Applications (ESA) is rather difficult and takes a long time while it costs a lot of money and introduces a lot of inconveniences within the company. In addition to this, the customization of such ESA is not easy either and sometimes it leads the enterprise to make important changes in its organization and which decreases its performances. The last but very important point is the issue of the interoperability. The aims of the INTEROP project were to harmonize and synthesize existing researches around new flexible and adaptive architectures of the interoperability, - such as the model driven approach, service-oriented architecture approaches, peer-2-peer architectures, agent architectures and federated architectures [12]. A P2P network is built of interconnected smaller networks which are the result of longrunning transactions corresponding to automated (B2B) business activities [15]. ERPs for SMEs do not have such wide of range functions as ERP II which is for a large organisation but many basic functions are necessary for the interoperability features with other SMEs in the DBE (Figure 4.). An example for creation of a DBE for the shoe domain which requires a reliable and secure communication infrastructure among SMEs describe by Chituc [1].

Interoperability is a multidimensional problem that can concern different layers of the enterprise. One of the difficulties enterprises are facing with is the lack of interoperability of systems and software applications which are managing progress in their business. Organizations are looking for new methods of work and business relationships, and the exchange of information and documents with new partners are often impossible to be executed automatically in an electronic format. The above mentioned 
problem is mainly caused by incompatibility in the information representation and in adopted software application methods [6]. Several approaches have been developed in order to achieve a collaborative network their focus, however, is mainly on technical aspects related to inter-organizational communication. The service-oriented architecture (SOA) as "a set of components which can be invoked, and whose interface descriptions can be published and discovered" does not take into consideration the service architecture. Technical interoperability concerns technical issues related to ecommunication, e.g., issues on linking applications and services addressing aspects related to: interfaces; ICT platforms; information integration; exchange and accessibility; security, standards; services but this is not enough to achieve the best solution in business applications. Information /knowledge interoperability has to focus on the following aspects: information/knowledge representation and management, learning ability, rights to access information, knowledge sharing, aspects related to adaptation and recombination of knowledge in a collaborative network during its life-cycle. The Framework for Enterprise Interoperability (FEI), which is currently under standardisation (CEN/ISO 11354) defines a classification scheme for the categorisation of knowledge for interoperability in the line of three dimensions: interoperability barriers, interoperability approaches, and enterprise levels [11]. The technical architecture of KodA is based on SOA and subsequently consists of three basic layers: business process management layer, business services layer, business application layer. The KodA focuses on the supply chain for processing food products which was communicated and discussed at different forums. This has resulted in establishing the agriXchange group that has the objective of harmonizing agricultural data exchange on a European level [17].

\section{A Solution Architecture and Possible Application Domains}

Applications based on DBE philosophy can be used in many places. As it can be seen in the figure (Fig. 3.) the data are stored in individual peers separately and there are DBE servers which require data from these peers. This structure of the data storage creates the possibility that all of the data owner store own data securely and the data are send only to an appropriate request by a duly authenticated server. This method is very advantageous in many ways.

DBE applications according to the Lisbon agreement [9] are developed for SMEs to help them in winning over the competition and stay on the market. Our solution is quite generic and it can be used in many domains wherever value chains can be developed and computer-aided business management exist. Usually one SME cannot cover the entire value chain as oppose to a multinational company. Joining to a value chain can be a breaking point for them. Nowadays the main field where the DBE philosophy is used is mainly knowledge sharing.

Fig. 2. shows that many master servers can be implied in the ecosystem and the peers can connect to them. 


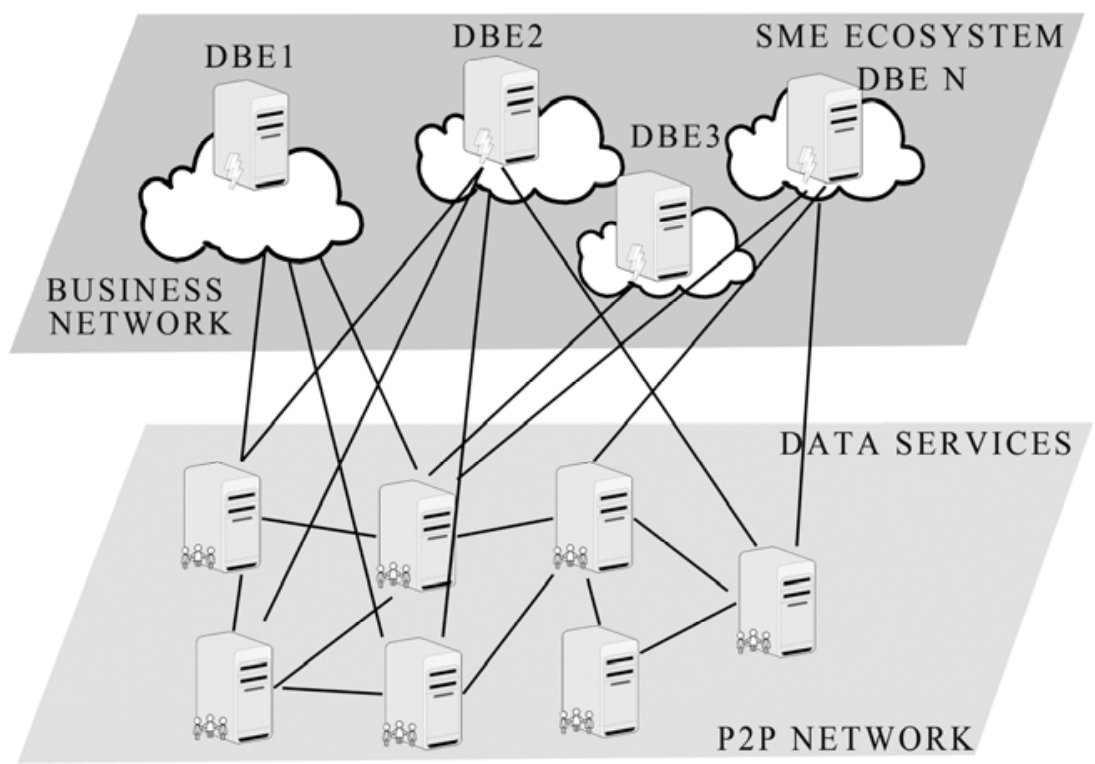

Fig. 2. The DBE concept

Since it is designed in a way that a peer can connect to more than one main server the data modified in one place (maintained) can be displayed in several places (servers). It is a cost friendly solution from the clien side, especially as the data can be managed, updated, modified, saved, etc in one place. Another advantage is if one or two peers are out from the network the ecosystem can work further. Contact between peers and servers are made by XML, so a peer can connect to any of this ready-made ERP for accepting the request to join.

With our prototype solution the SMEs can provide safe, transparent, direct services to potential customers. According to the Client-Server architecture, all data are stored on a dedicated server. This can provide great advantages for the server's owner. Our vision is based on P2P SOA technology and all components are written with Open Source tools. The main features of the prototype system are the following.

- The first idea was to try to create a general service flowchart.

- Next if you needed a service probably you would need other ones from the flowchart. (Example: If you booked a room somewhere next week, probably you would like to use the public transport or would like to know the theatre program, or the time-tables, etc.).

- The prices of services, unique features, or discounts, etc. are not stored on the server.

- An adequate identification system will be worked out.

- Our aim is not to cover all the business processes, but to model a new procedure.

- Many processes are similar to each other therefore the system has to suggest a join possibility for the Peer. 
- Since in many cases the SME-s especially the Micro enterprises have no adequate expert, we have to create an easily manageable layout.

- Open source software is used.

- Easy and friendly development of services and application interfacing between companies;

- Easy publishing of the services and applications, including the relevant information,

- Utilization of combined services and applications, in heterogeneous forms.

- In multi-agent systems, the underlying networks in a Digital Business Ecosystem are always dynamic and network topologies are always changing over the time. The main server is always able to work, but the other habitats sometimes are up or down. The P2P communications are working via OWL, WSDL, XML, RDF technologies (Fig. 3.). It can be seen that after a query the system process the query and the BPE try getting up what we want (If it has enough experience about it) or the adequate informations are stored in the knowledge base. Afterwards it collects all the necessary data and it compose the result. All movement and arose data are stored in the database for further utilization.

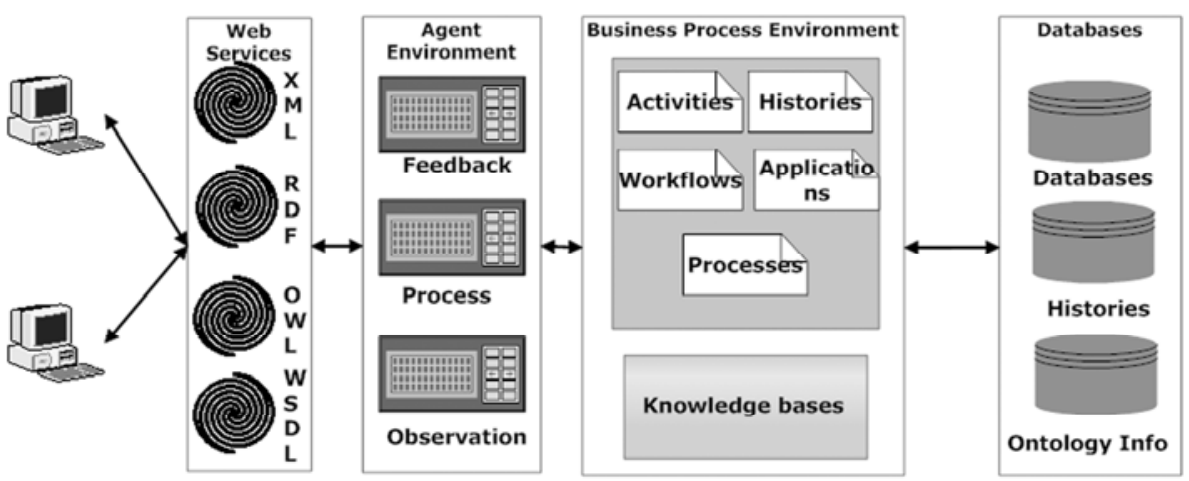

Fig. 3. Layered model of the prototype

XML Web services are the fundamental building blocks in the move to distributed computing on the Internet. Open standards and the focus on communication and collaboration between people and applications have created an environment where XML Web services are becoming the platform for application integration. Applications are created using multiple XML Web services from various sources that work together regardless of where they are or how they were implemented.

- XML Web Services provide useful functionality to Web users through a standard Web protocol. In most cases, the used protocol is SOAP.

- XML Web services provide a solution to describe their interfaces which enough detail to allow a user to build a client application to talk to them. This description is usually provided in an XML document called a Web Services Description Language (WSDL) document.

- XML Web services are registered so that potential users can find them easily. This is done with Universal Discovery Description and Integration (UDDI). 
The above mentioned technologies are feasible to connect different systems. The interface can be adapted in the various systems for real-time interface connection. The development is related to DBE marketplace testing of modules are in progress (Table 1).

Table 1. Main parts of our model

\begin{tabular}{|l|l|}
\hline NAME & DESCRIPTION \\
\hline $\begin{array}{l}\text { Knowledge } \\
\text { Base (KB) }\end{array}$ & $\begin{array}{l}\text { The function of this module is to collect information with along the } \\
\text { community principles }\end{array}$ \\
\hline $\begin{array}{l}\text { Service } \\
\text { Generator } \\
(\mathrm{SG})\end{array}$ & $\begin{array}{l}\text { Its function is to analyse the requirements, generate the workflow } \\
\text { and collect relevant information from the provider. }\end{array}$ \\
\hline $\begin{array}{l}\text { Relevance } \\
\text { Generator } \\
(\text { RG) }\end{array}$ & $\begin{array}{l}\text { It tries to generate a relevance number from the tabs, queries, and } \\
\text { stored history }\end{array}$ \\
\hline $\begin{array}{l}\text { Message } \\
\text { System } \\
(\mathrm{MS})\end{array}$ & It is a private and secured mailing system \\
\hline $\begin{array}{l}\text { History Base } \\
\text { (HB) }\end{array}$ & $\begin{array}{l}\text { The function is to store and process the requirements, data, tabs, } \\
\text { etc. }\end{array}$ \\
\hline
\end{tabular}

Given our research project is rather complex; we decided to split it into parts. The main advantage of our approach is that each program module works alone. We defined the input and output of each part, but we didn't define how to reach it. So in the prototype there is a possibility of changeing parts.

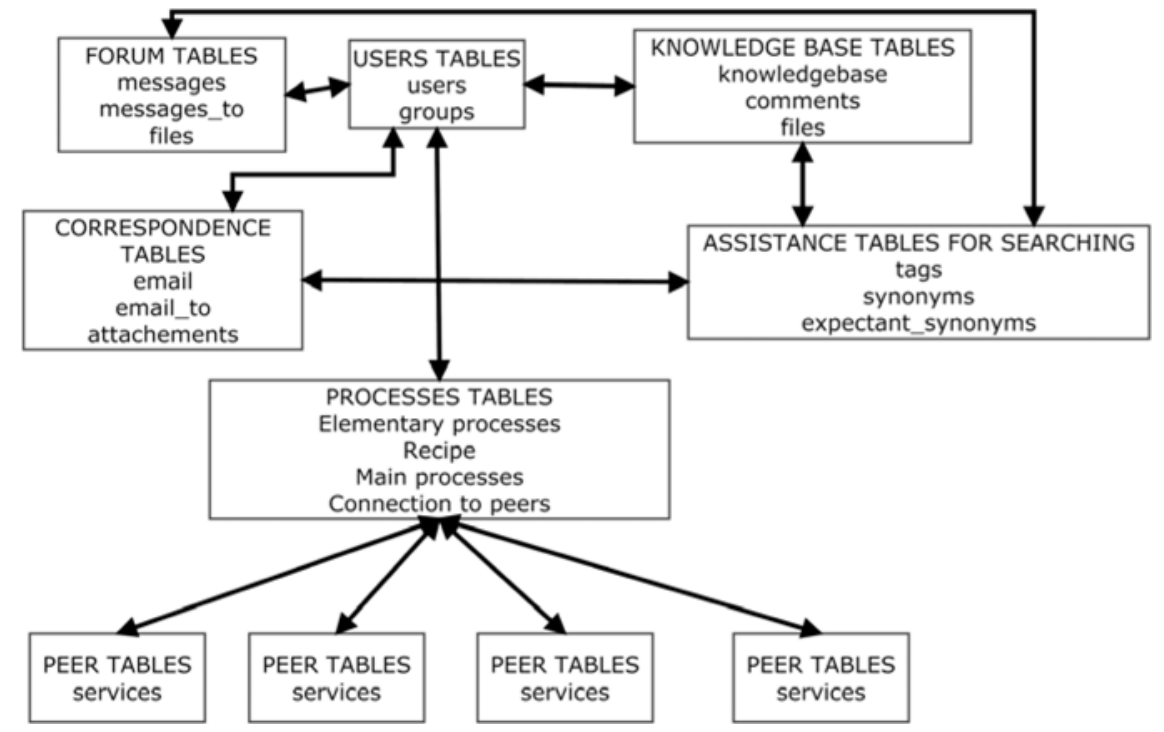

Fig. 4. Layered model of the prototype 
Fig. 4. shows the structure of the database and as it can be seen in the portal it is made up of modules. Some modules are compulsory (example user or log), and others are optional. The peer tables are installed on the peers. The peer-specific offerings are stored in these tables, which are downloaded to the portal after the site selection process. The other tables are located in the main server (portal). These tables help to communicate, sharing the knowledgebase, blogging, etc. and store the coherence as well. Besides of these the main task of the server database is to store the new arosen information. This information can help for the portal becoming better. The database is placed in a MySQL server because it is easy to use and free.

We believe, it is worth to capitalize the potential of the DBE philosophy in much more fields. Our prototype can assist that kind of businesses which are able to join to any value chain. For instance the typical good application area is the tourism management, especially the destination management.

\subsection{Applications in Tourism}

Many SMEs who work variety of business activity can join to the value chains in tourism sector. These SMEs can create value chains according to community principles and find our places within the value chain. Additional services make the site to become more useful for the members of the community and for the potential costumers as well. The different types of knowledge basis which are characterized by meta-data attached to each other and to adequate part of the value chain as well, thus extend the usefulness of the portal. Because the most of enterprises already use different ERP system or at least their data are stored somehow. To get out the problem of the duplicate storing, we offer the opportunity supplying the data from their own database with the help of XML technology. With the XML technology the companies without any particular investment can join to the DBE community and they can find new markets, knowledge and community.

\subsection{A Prototype Tracing Solution Based on DBE Consept and Toolset}

IT solutions of the food chain traceability could be based on DBE solutions. In case of the traceability the XML file contains the following information: Company name, TRU (Traceable Resource Unit) identification number, Output id., Input id. In addition, we need a web server where the portal software is running. The portal prepaired with open source tools according to DBE principles. The prototype system is suitable for both top-down and bottom-up tracking and traceing. The working methods are the follows:

Top-down: The web server can identify the producer by the barcode. Certainly, it works only if the company has joined the community and their barcodes are stored in the database. We can reach the data wich are strored in the ERP system by the product's barcode. The data show us the ingredients of the product. Inputs displayed on this page, so that the input supplier of the database searches the details of ingredients and send to the server for further processing and display, and then recursively to the product suppliers to get the similar information. We can reach the bottom level of the supply chain. It looks like a tree-structure.

Bottom-up: The knowledge of the barcode of the lowest level of basic ingredient we can get besides of the basic details we can know the place of the deliveries as well. 
Then, either of the products manufactured by the companies which are on the N-1st level will be choosen which contain the ingredient of the company on the Nth. level. This goes on, until we reach the top level.

With this prototype we have an opportunity to trace the full path of life of the product, if only all participant have been joined to the community. The above solution can greatly facilitate the precise monitoring the flow of substances occurring in food. Thus, the appearance of any food safety hazard we have opportunity to achieve rapid and efficient product recall.

\section{Conclusions}

The concept of Digital Business Ecosystem (DBE) is to build an Internet-based environment in which businesses are able to interact with each other efficiently. In the near future when this platform will be realized, it will make it possible to Small and Medium Enterprises (SMEs) to compete on the market with equal opportunities, independently of size and distance from the city, of their business. The first characteristic of an ecosystem is that business ecosystems have a loose network of suppliers, distributors, outsourcing firms, makers of related products or services, technology providers, and a host of other organizations. The second characteristic is the of "platform" - services, tools, or technologies - that other members of the ecosystem can use to enhance their own performance. The third characteristic is that, business ecosystem evolves participants to a new landscape [7]. The Open source approach is the only possible choice for the Digital Ecosystem infrastructure [3]. We created our own vision which is able to help the SMEs to appear on the market while protecting its own data. The communication interface allows connecting the existing ERP systems into one DBE cluster. The use of the suggested security tool helps to keep authenticity of the companies.

\section{References}

1. Chituc, C.-M., Toscano, C., Azevedo, A.: Towards the Creation of a Digital Business Ecosystem for the Shoe Manufacturing Domain. In: Digital EcoSystems and Technologies Conference, DEST (2007), Inaugural IEEE-IES 88 - 93, doi:10.1109/DEST.2007.371951

2. Digital Business Ecosystem Contract n 507953: DBE Peer-to-Peer Architecture Design. TCD, DBE Deliverable, D24.3 - Final version of the P2p architecture for service search (2005)

3. European Commission. Digital Business Ecosystem. Luxembourg Office for Official Publications of the European Communities (2007), ISBN 92-79-01817-5

4. Adomavicius, G., Bockstedt, J.C., Gupta, A., Kauffman, R.J.: Technology roles and paths of influence in an ecosystem model of technology evolution. In: LLC 2007. Inf. Technol. Manage., February 14, vol. 8, pp. 185-202. Springer Science+Business Media, Heidelberg (2007), doi:10.1007/s10799-007-0012-z

5. IEEE: IEEE Standard Computer Dictionary: A Compilation of IEEE Standard Computer Glossaries. Institute of Electrical and Electronics Engineers, NY (1990)

6. Jardim-Goncalves, R., Grilo, A., Steiger-Garcao, A.: Challenging the interoperability between computers in industry with MDA and SOA Computers in Industry 57(8-9), 679-689 (2006) 
7. Li, Y.: The technological roadmap of Cisco's business ecosystem. Technovation 29, 379386 (2009)

8. Moore, J.F.: Predators and Prey: A New Ecology of Competition. Harvard Business Review 71(3), 75-85 (1993)

9. Nachira, F.: Towards a Network of Digital Business Ecosystems Fostering the Local Development. European Commission DG INFSO, Tech. Rep., pp. 1-23 (2002)

10. Nachira, F.: Innovation Ecosystems: Una Strategia europea per l'innovazione e lo sviluppo economic (2005), http://www.digital-ecosystems.org (retrieved August 21, 2006)

11. Naudet, Y., Latour, T., Guedria, W., Chen, D.: Towards a systemic formalisation of interoperability. Computers in Industry 61(2), 176-185 (2010)

12. Panetto, H., Scannapieco, M., Zelm, M.: INTEROP NoE Interoperability Research for Networked Enterprises Applications and Software. In: Meersman, R., Tari, Z., Corsaro, A. (eds.) OTM-WS 2004. LNCS, vol. 3292, pp. 866-882. Springer, Heidelberg (2004)

13. Pappas, N., Kazasis, F.G., Anestis, G., Gioldasis, N., Christodoulakis, S.: A Knowledge Management Platform for Supporting Digital Business Ecosystems based on P2P and SOA technologies. In: IEEE DEST 2007 (2007)

14. Peltoniemi, M., Vuori, E.: Business ecosystem as the new approach to complex adaptive business environments. In: eBRF Conference: Frontiers of e-Business Research (2004)

15. Razavi, A., Moschoyiannis, S., Krause, P.: An open digital environment to support business ecosystems. In: Peer-to-Peer Netw. Appl. Springer Science + Business Media, LLC 2009 (2009), doi:10.1007/s12083-009-0039-5

16. Szerb, L.: The connection between innovation and business growth in the Hungarian SME sector. In: Innovation, Competitiveness, Growth and Tradition in SMEs, Rencontres de StGall, September 1-3 (2008)

17. Wolfert, J., Verdouw, C.N., Verloop, C.M., Beulens, A.J.M.: Organizing information integration in agri-food-A method based on a service-oriented architecture and living lab approach. Computers and Electronics in Agriculture 70(2), 389-405 (2010) 